Eur. J. Clin. Chem. Clin. Biochem.

Vol. 30, 1992, pp. 737-744

(C) 1992 Walter de Gruyter \& Co.

Berlin · New York

\title{
Alteration of the Permeability of the Human Erythrocyte Membrane to Cations by Liposome-Incorporated Amphotericin B
}

\author{
By K. M. Abu-Salah \\ Department of Biochemistry, College of Science, King Saud University, Riyadh, Saudi Arabia
}

(Received December 5, 1991/May 22, 1992)

\begin{abstract}
Summary: Some effects of liposome-incorporated amphotericin B on the permeability of the human erythrocyte membrane to potassium and sodium ions is reported. The influence of cholesterol and amphotericin B in causing a shift towards smaller and larger liposomes, respectively, is also described. Phosphatidylcholine liposomes containing amphotericin B in a molar ratio of $7.4 \pm 0.1$ (mean \pm SD) antibiotic to 1000 phospholipid reduced the initial rates of $\mathrm{K}^{+}$and $\mathrm{Na}^{+}$transport across the erythrocyte membrane to $40 \pm 2.6 \%$ and $0 \%$, respectively, of their rates in the presence of comparable concentrations of free amphotericin $\mathrm{B}$. Amphotericin B incorporated into liposomes $(8.2 \pm 0.15 \mu \mathrm{mol}$ antibiotic per $1000 \mu \mathrm{mol}$ total lipid) composed of cholesterol and phosphatidylcholine (in a molar ratio of $3: 7$ ) reduced the initial rate of $\mathrm{K}^{+}$transport to $19 \pm 0.8 \%$ of its value measured in the presence of a comparable concentration of free antibiotic. These results suggest that liposomes containing specified amounts of amphotericin $\mathrm{B}$, especially liposomes also containing cholesterol in addition to phosphatidylcholine, could be used as a method of controlling $\mathrm{K}^{+}$ transport across the erythrocyte and possibly other types of cellular membranes, thereby limiting antibiotic toxicity to some mammalian tissues.
\end{abstract}

\section{Introduction}

Amphotericin B and nystatin are highly effective, but toxic, polyene macrolide antibiotics, used in the treatment of a wide range of fungal infections (1). They achieve their fungicidal and lytic activities by complexing with the sterols available in plasma membranes $(2-4)$, thus forming transmembrane channels, which allow the efflux of vital cations such as potassium $(4,5)$. They inhibit, however, the exchange of some metabolically important anions such as phosphate and sulphate $(6,7)$. In addition, the formation of channels leads to disruption of sterol-phospholipid interactions in membranes (8). These combined effects of this class of ionophoric antibiotics lead eventually to cell lysis and death. The ability of intravenously administered amphotericin B to form channels by interaction with cholesterol in mammalian plasma membranes (4), but with a lower affinity than with fungal ergosterol $(4,28)$, could be the basis for its haematopoietic, nephrotic and central nervous system toxicities $(9-11)$. Since amphotericin B is the drug of choice for most systemic mycoses, arising as complications in patients with haematological malignancies $(12,13)$ and in immunocompromised patients $(14$, $15)$, attempts have been made to reduce its toxicity and to improve its therapeutic efficacy by incorporating it into liposomes prior to its intravenous injection $(16-20)$.

Multilamellar liposomes $(21-23)$ have been used as carriers of antimicrobial (24), antiparasitic (25) and antineoplastic drugs (26) to improve their therapeutic index. Liposome-incorporated amphotericin B has been studied in a variety of experimental infections. New et al. (16), for example, showed that liposomeincorporated amphotericin B was less toxic and more active against experimental Leishmaniasis than the free drug. A lower toxicity and an equal therapeutic efficacy were observed by Taylor et al. (18) against experimental histoplasmosis, by Graybill et al. (17) 
against experimental cryptococcosis, and by LopezBerestein (27) and Lopez-Berestein et al. (19, 20) against experimental candidiasis.

The purpose of this study is to investigate the possible reduction of cell damage and toxicity toward human erythrocytes, but with obvious implications for other cell types, by using liposome-incorporated polyene antibiotics in conjunction with or without free therapeutic steroid derivatives. In this work, liposomes were used as carriers for specified amounts of amphotericin B or nystatin.

\section{Materials and Methods}

\section{Materials}

Human blood, with added standard citrate-dextrose-adenine solution, was kindly supplied by the blood bank of King Khalid University Hospital, Riyadh. Amphotericin B was obtained from Sigma Chemical Co., Kingston upon Thames, Surrey, U.K. Nystatin and ouabain were purchased from Windsor Laboratories, Slough, Berkshire, U.K. $L$ - $\alpha$-Phosphatidylcholine from egg yolk was provided by Calbiochem Corporation, San Diego, U.S.A. Cholesterol was supplied by Riedel-de Haen, Hannover, Germany. Digitoxin and digitoxigenin were obtained from BDH Chemicals, Poole, Dorset and Koch-Light Laboratories, Colnbrook, Bucks, U.K., respectively. Hydrocortisone sodium succinate in its clinical formulation was a product of Upjohn S. A., Puurs, Belgium. All other reagents were analytical grade or better and were obtained from $\mathrm{BDH}$ Chemicals.

\section{Methods}

\section{Preparation of liposomes}

A solution of phosphatidylcholine in methanol : chloroform ( $2: 1$, by vol.), was evaporated to dryness under nitrogen. The resulting pellet was suspended in an isotonic solution of $9 \mathrm{~g} / \mathrm{l}$ $\mathrm{NaCl}$ by hand shaking for a few minutes to form liposomes. Liposomes containing cholesterol were prepared by the same method at a molar ratio of cholesterol to phospholipid of $3: 7$. Liposomes prepared in this way were sedimented by centrifugation at $700 \mathrm{~g}$ in a MSE Coolspin centrifuge, then suspended in Tris-buffered saline $(5 \mathrm{mmol} / 1$ Tris, $4 \mathrm{mmol} / 1 \mathrm{KCl}, 146 \mathrm{mmol} / \mathrm{l}$ $\mathrm{NaCl}, \mathrm{pH}$ 7.4). The amounts of cholesterol and phospholipid in the liposomes were estimated by determining the amounts of these constituents in both the supernatant and the pellet. The size distribution of the prepared liposomes was determined using an electronic particle size analyser (Coulter Counter, model ZM). This device simultaneously enumerates the number of particles in each of sixteen different sizes based on average volume.

\section{Incorporation of antibiotics into liposomes}

Prior to incorporation, antibiotics were sonicated in $9 \mathrm{~g} / 1 \mathrm{NaCl}$ for $2 \times 10 \mathrm{~s}$ intervals on ice using a MSE Soniprep 150 at an amplitude of $10 \mu \mathrm{m}$. An aliquot of the antibiotic preparation was taken prior to and after sonication to determine the size distribution of antibiotic particles as described above. Sonicated antibiotic suspensions were incubated with liposomes in Tris buffered saline at a molar ratio of antibiotic to liposomal phospholipids of 1 to 100 for $25 \mathrm{~min}$ at room temperature. To estimate the amount of antibiotic incorporated into liposomes, the unincorporated particulate amphotericin B or nystatin was separated from liposomes by centrifuging the antibiotic lipid mixtures for $10 \mathrm{~min}$ at $500 \mathrm{~g}$. The quantity of antibiotic incorporated into liposomes was estimated by dissolving the pellet, after washing it once with Tris buffered saline, in methanol : chloroform ( $2: 1$, by vol.), then measuring the absorbance at $405 \mathrm{~nm}$ and $303 \mathrm{~nm}$ for amphotericin $B$ and nystatin, respectively, using pure antibiotics as standards. Phospholipid and cholesterol in the supernatants were determined by the methods of Chen et al. (29) and Leffler \& McDougal (30), respectively. The size distribution of liposome-incorporated antibiotic was determined as described above.

Measurement of potassium and sodium transport in erythrocytes treated with liposome-incorporated antibiotics

Erythrocytes were prepared by washing normal human blood in Tris buffered saline and centrifuging at $1000 \mathrm{~g}$ for $10 \mathrm{~min}$ at $4{ }^{\circ} \mathrm{C}$. After removal of the buffy coat, the erythrocytes were washed twice again with Tris buffered saline as above. Washed erythrocytes were pre-incubated with liposomes containing the antibiotic, or with the free antibiotic solubilized in dimethyl sulphoxide, using the same antibiotic concentration in both cases. Pre-incubation was carried out for $15 \mathrm{~min}$ in Tris buffered saline at a haematocrit value of 0.45 and $37^{\circ} \mathrm{C}$, in the presence or absence of digitoxin, digitoxigenin, or hydrocortisone succinate. Controls were performed in the presence or absence of free antibiotic, and with empty liposomes plus free cholesterol or free phosphatidylcholine.

In order to measure sodium and potassium transport, samples were removed from the reaction medium at various time intervals and centrifuged at $2000 \mathrm{~g}$ for $0.5 \mathrm{~min}$. Aliquots were taken from the supernatant for haemoglobin determination using a Boehringer Mannheim haemoglobin kit. Further aliquots of the supernatant were taken and subsequently diluted with Tris buffered saline for $\mathrm{K}^{+}$determination, using a Dr. Lange M7D flame photometer. $\mathrm{Na}^{+}$was determined with a Pye Unicam SP9 atomic absorption spectrophotometer, in aliquots of supernatants diluted with $6 \%$ isobutanol. All concentrations of the antibiotic, together with a control containing dimethyl sulphoxide where necessary, were examined in duplicate in one experiment.

\section{Results}

\section{Effect of cholesterol on liposomal size distri-} bution

The size distribution of the prepared liposomes was assessed using a Coulter Counter, model ZM. The average diameter of about $95 \%$ of the phosphatidylcholine liposomes and those composed of both cholesterol and phosphatidylcholine was less than $2.88 \mu \mathrm{m}$ (fig. 1a). However, the total number of phosphatidylcholine liposomes containing cholesterol was $8.3 \pm 0.6 \%$ (mean $\pm \mathrm{SD}$ ) less than that of phosphatidylcholine liposomes lacking cholesterol. According to the phospholipid assay, there was no increase in the residual amount of free un-incorporated phosphatidylcholine molecules when liposomes were formed from cholesterol and phosphatidylcholine in a molar ratio of $3: 7$. The volume for each average liposome diameter was calculated from the frequency data, which are given in figure 1a. Total volume distribution analysis (fig. 1b) showed that the volume of cholesterol-containing liposomes was $19 \pm 1.3 \%$ 

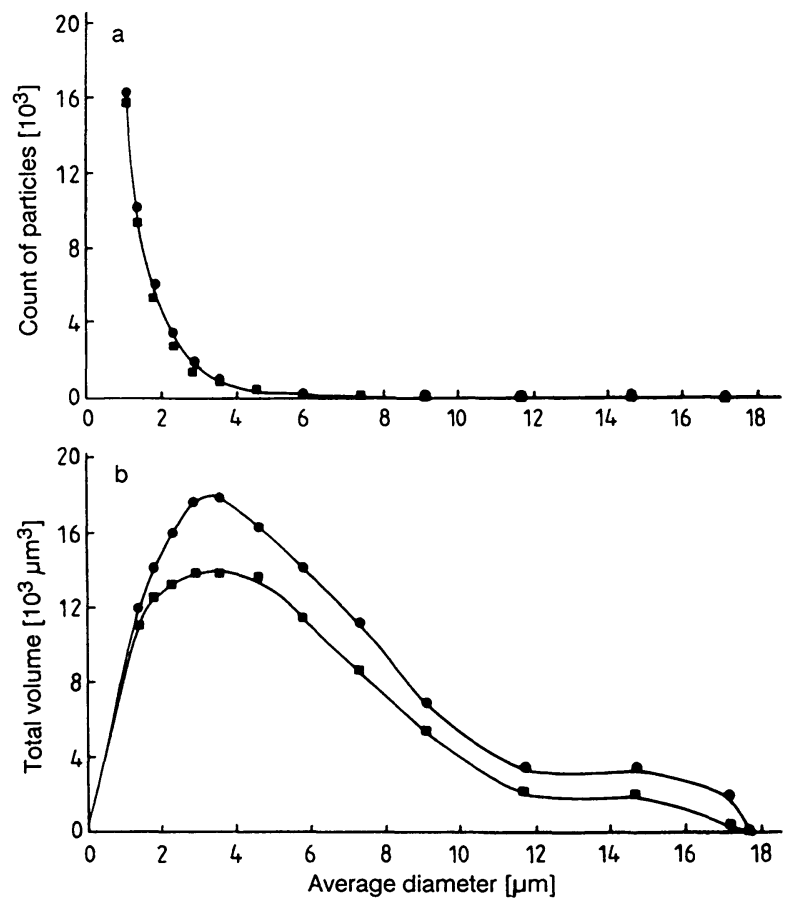

Fig. 1. Effect of cholesterol on liposomal average diameter distribution. Liposomes composed of phosphatidylcholine $(\bullet)$ or cholesterol and phosphatidylcholine ( $\boldsymbol{(})$ at a molar ratio of $3: 7$. The size distribution of the liposomes was determined using an electronic particle size analyser (Coulter Counter, model ZM) at a manometer volume of $50 \mu \mathrm{l}$, and aperture resistance of $33.6 \mathrm{k} \Omega$, a pre-set gain of 1.0 , a calibration factor of 5.3 , a calibration diameter of $1.054 \mu \mathrm{m}$ and a calibration volume of $0.6036 \mu^{3}$. Diameter calibration was performed using latex particles, number 9316007 (Coulter electronics), using an aperture diameter of $30 \mu \mathrm{m}$, which spanned a total diameter range of $0.6-18 \mu \mathrm{m}$. The data shown correspond to $250 \mu \mathrm{l}$ of liposomes containing 63 $\mu \mathrm{mol}$ of liposomal phosphatidyl-choline or $19 \mu \mathrm{mol}$ of liposomal cholesterol and $44 \mu \mathrm{mol}$ of phosphatidylcholine. Liposomes were diluted $800 \times$ with $0.4 \mathrm{mmol} / 1$

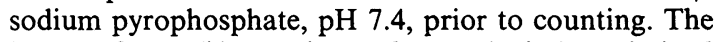
bottom frame (b) was drawn from calculations derived from the data shown in the top frame (a) as described in Materials and Methods. Each point represents the mean of five different determinations.

less than that of liposomes lacking cholesterol, presumably partly due to the smaller total number of liposomes of the former type. Particles larger than $2.88 \mu \mathrm{m}$ in diameter are expected to be mainly aggregates of multilamellar liposomes.

\section{Size distribution of amphotericin B particles}

The availability of particles of amphotericin B, and their size distribution in Tris buffered saline, was demonstrated using a Coulter Counter, model ZM (fig. 2). The average diameter of more than $95 \%$ of amphotericin B particles, prepared from sonicated or unsonicated medium, was less than $3.45 \mu \mathrm{m}$ (fig. 2a). The particles counted are most probably aggregates of micelles, as individual micelles are too small to be
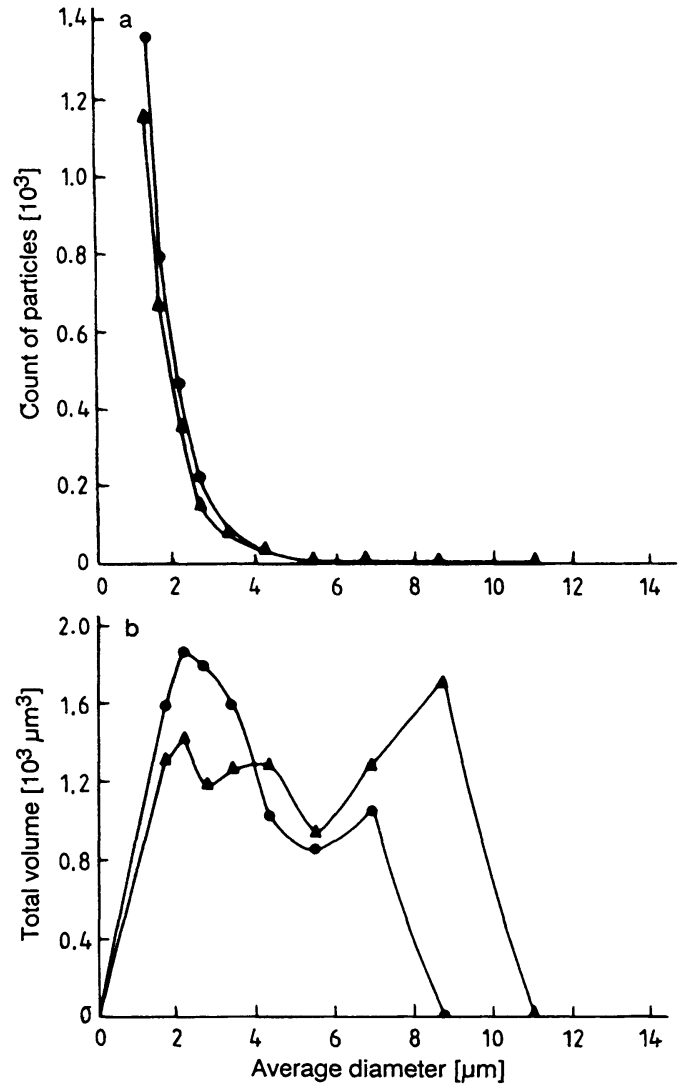

Fig. 2. The effect of sonication on size distribution of amphotericin B particles. The average diameter distribution of amphotericin B was measured under conditions similar to those described in the legend of figure $1 \mathrm{a}$ except that a pre-set gain of 2.0 , a calibration factor of 6.35 , a calibration volume of $2.1 \mu \mathrm{m}^{3}$ and an aperture resistance of $19.3 \mathrm{k} \Omega$ were used. Diameter calibration was performed using latex particles, number 9316008 using an aperture diameter of $50 \mu \mathrm{m}$ which spanned a total range of 0.8 to $30 \mu \mathrm{m}$. The data shown correspond to $250 \mu \mathrm{l}$ of $10 \mu \mathrm{mol} / 1$ stock of sonicated $(\bullet)$ or unsonicated $(\boldsymbol{\Delta})$ amphotericin B. The amphotericin B sample taken was diluted $400 \times$ with $0.4 \mathrm{~mol} / 1$ sodium pyrophosphate, $\mathrm{pH}$ 7.4 , prior to counting. The bottom frame (b) was drawn from calculations derived from the data shown in the top frame (a) as described in Materials and Methods. Each point represent the mean of four different determinations.

quantitated by this method. The total number of amphotericin B particles was increased by $22 \pm 1.7 \%$ upon sonication. The volume at each average diameter of amphotericin B particles was calculated from the frequency data which are given in figure 2a. Total volume distribution analysis (fig. $2 b$ ) showed that sonication of amphotericin B dispersions led to the formation of a less heterogeneous population of particles (fig. 2b). Moreover, statistical analysis, in which the data of figure $2 b$ were summated, showed that sonication of amphotericin B dispersions caused a shift toward smaller particles. In spite of their higher total count, sonicated amphotericin B particles had about the same total volume as those of unsonicated ones. 
Incorporation of amphotericin B into liposomes and its effect on liposomal size distribution

To achieve optimal conditions for incorporating amphotericin B or nystatin into liposomes, antibiotic dispersions were sonicated, then incubated with unsonicated liposomal preparations. The unpartitioned antibiotic portion was separated from antibioticloaded liposomes at low centrifugal force. The centrifugation of mixtures of sonicated amphotericin B and sonicated liposomes, or unsonicated amphotericin B and unsonicated liposomes did not efficiently separate the un-incorporated portion of the antibiotic from the liposome-incorporated antibiotic. The molar ratio of incorporated antibiotic to liposomal lipid was found to be $7.4 \pm 0.1 / 1000$ and $8.2 \pm 0.15 / 1000$ in phosphatidylcholine and cholesterol liposomes, respectively. Much lower percentages of nystatin were found to be incorporated into liposomes. The molar ratio of antibiotic to membrane lipid was found to be $2.5 \pm 0.1 / 1000$ and $3.0 \pm 0.12 / 1000$ in phosphatidylcholine and phosphatidylcholine cholesterol liposomes, respectively.

The average diameter distribution of liposomes containing amphotericin B (fig. 3a) revealed that the average diameter of more than $95 \%$ of liposomes, containing or lacking cholesterol, was less than 3.6 $\mu \mathrm{m}$. The volume at each average liposome diameter was calculated from the frequency data given in figure 3a. Total volume distribution analysis, in which the data of figure $3 b$ were summated (fig. $3 b$ ), showed that the incorporation of amphotericin B into cholesterol-containing liposomes caused a shift toward larger liposomes. Although their total count was decreased by $9.7 \% \pm 1.7 \%$, the total volume of these liposomes was $14.6 \pm 1.1 \%$ greater than that of cholesterol-lacking liposomes loaded with amphotericin B (fig. 3b).

Permeability of the human erythrocyte membrane to cations under the influence of liposome-incorporated amphotericin B

Incorporation of sonicated amphotericin B into phosphatidylcholine liposomes at a molar antibiotic to phospholipid ratio of 7.4 to 1000 reduced the initial rates of $\mathrm{K}^{+}$and $\mathrm{Na}^{+}$transport across the erythrocyte membrane to $40 \pm 2.6 \%$ (fig. $4 \mathrm{a}$ ) and $0 \%$ (fig. $4 \mathrm{~b}$ ), respectively, of their rates in the presence of comparable concentrations of free amphotericin B. Despite a more efficient incorporation of amphotericin $B$ into liposomes $(8.2 \mu \mathrm{mol}$ antibiotic per $1000 \mu \mathrm{mol} \mathrm{lipid})$, at a molar ratio of cholesterol to phosphotidylcholine of $3: 7$, the initial rate of $\mathrm{K}^{+}$transport was reduced

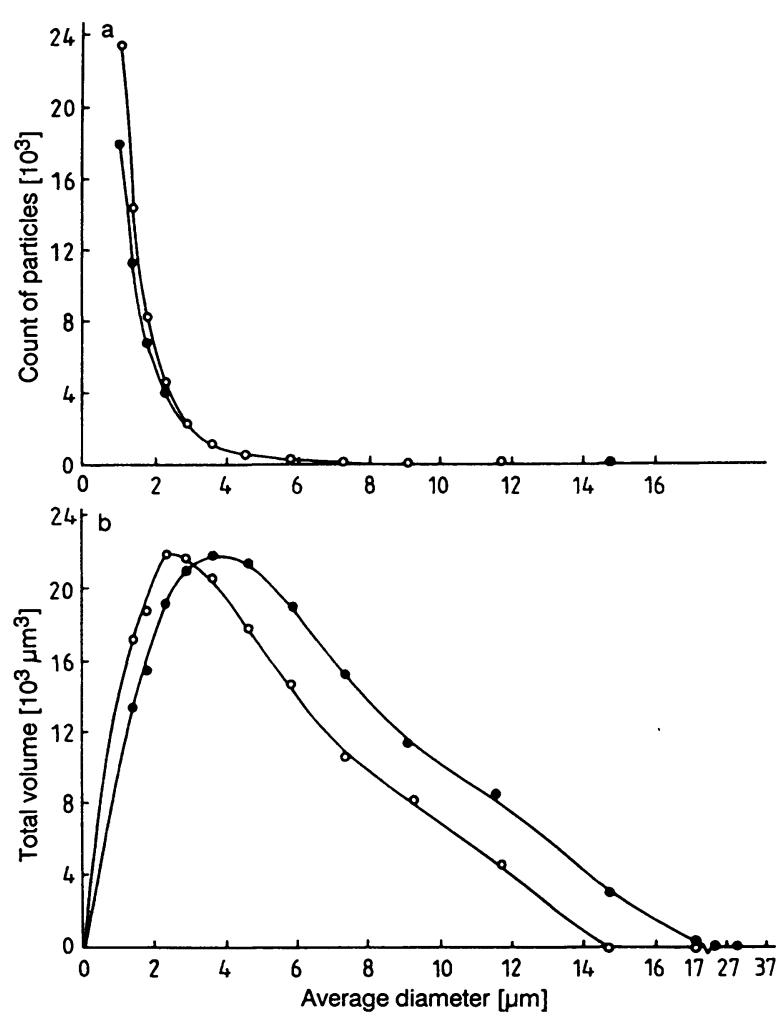

Fig. 3. Effect of amphotericin B on the average diameter distribution of liposomes. The conditions for determining the average diameter distribution were the same as those described in the legend of figure 1a. The data shown correspond to $250 \mu \mathrm{l}$ of amphotericin B incorporated in $63 \mu \mathrm{mol}$ of liposomal phosphatidylcholine (O) or 19 $\mu \mathrm{mol}$ of cholesterol and $44 \mu \mathrm{mol}$ phosphatidylcholine (๑). Liposomes were diluted $400 \times$ with $0.4 \mathrm{~mol} / 1$ sodium pyrophosphate, $\mathrm{pH} 7.4$, prior to counting. The bottom frame (b) was drawn from calculations derived from the data shown in the top frame (a) as described in Materials and Methods. Each point represents the mean of six different determinations.

to $19 \pm 0.8 \%$ of its value with a comparable concentration of free antibiotic (fig. 4a). The effects of incorporating higher concentrations of amphotericin $\mathrm{B}$ into liposomes, on $\mathrm{K}^{+}$and $\mathrm{Na}^{+}$transport, were not easy to assess. This was because the incubation of liposomes, even with sonicated antibiotic preparations of more than $10 \mu \mathrm{mol} / \mathrm{l}$ concentration, led to a partial precipitation of the un-incorporated antibiotic portion with liposomes, thus preventing an accurate assessment of the amount of amphotericin B incorporated into the liposomes. Potassium and sodium ion transport were further investigated by co-incubating antibiotic-free phosphatidylcholine cholesterol liposomes and free cholesterol, to check that the efficacy of cholesterol-containing liposome preparation, in reducing $\mathrm{K}^{+}$transport, was not due to an interaction between amphotericin $\mathrm{B}$ and un-incorporated cholesterol which might be attached to the surface of liposomes during their formation. The initial rates of transport of $\mathrm{K}^{+}$or $\mathrm{Na}^{+}$, however, did not seem to 

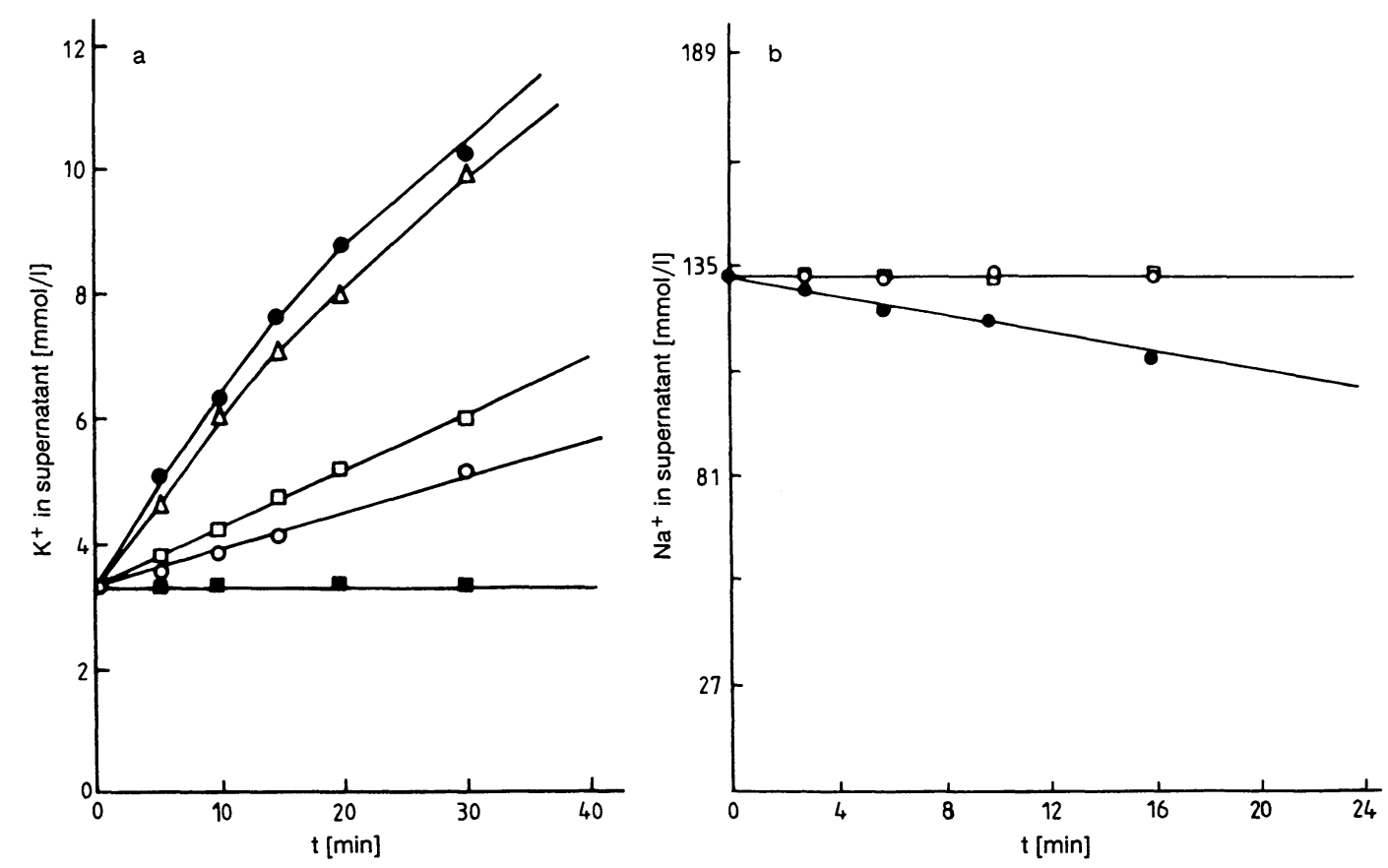

Fig. 4. a. Effect of liposome-incorporated amphotericin $\mathrm{B}$ on the rate of $\mathrm{K}^{+}$efflux. Washed human erythrocytes were incubated at $37^{\circ} \mathrm{C}$ with $0 \mu \mathrm{mol} / 1(\square), 8.1 \mu \mathrm{mol} / 1(\bullet)$ or $7.4 \mu \mathrm{mol} / 1(\triangle)$ free amphotericin B; amphotericin B, incorporated in 1000 $\mu \mathrm{mol}$ of liposomal phosphatidylcholine at a concentration of $7.4 \mu \mathrm{mol} / 1$, in the final suspension ( $\square$ ), amphotericin B, incorporated in $1000 \mu \mathrm{mol}$ of liposomal cholesterol and phosphatidylcholine at a molar ratio of $3: 7$, at a concentration of $8.1 \mu \mathrm{mol} / 1$ in the final suspension (O). The rate of $\mathrm{K}^{+}$effect was then monitored for $30 \mathrm{~min}$.

b. Effect of liposomes containing amphotericin $\mathrm{B}$ on the rate of $\mathrm{Na}^{+}$influx. Washed human erythrocytes were incubated at $37^{\circ} \mathrm{C}$ with $8.1 \mu \mathrm{mol} / 1$ free amphotericin B (•), amphotericin B incorporated in $1000 \mu \mathrm{mol}$ of liposomal phosphatidylcholine at a concentration of $7.4 \mu \mathrm{mol} / 1$ in the final suspension (O), or amphotericin B incorporated in $1000 \mu \mathrm{mol}$ of liposomal cholesterol and phosphatidylcholine at a molar ratio of $3: 7$, at a concentration of $8.1 \mu \mathrm{mol} / 1$ in the final suspension ( $\square$ ). The rate of $\mathrm{Na}^{+}$effect was then monitored for $16 \mathrm{~min}$. Each point represents the mean of five different studies.

be affected by mixing free cholesterol with preformed phosphatidylcholine cholesterol liposomes, at a molar ratio of cholesterol to total liposomal lipids of $5: 100$. In addition, neither antibiotic-free phosphatidylcholine liposomes, $12.5 \mathrm{mmol} / \mathrm{l}$ free phosphatidylcholine or $1.5 \%$ dimethyl sulphoxide caused any haemolysis or had any effect on the rates of $\mathrm{K}^{+}$or $\mathrm{Na}^{+}$transport. On the other hand, the efficacy of phosphatidylcholine, or cholesterol-phosphatidylcholine $(3: 7)$ liposomes, in minimizing the initial rate of cation exchange observed with free antibiotic, was reduced in the presence of free steroidal drugs, such as digitoxigenin, by $41 \pm 3.7 \%$ and $22 \pm 2.5 \%$ respectively (fig. $5 a)$. Values in the order of $57 \pm 4.1 \%$ and $16 \pm 1.1 \%$ were obtained with digitoxin under the same conditions (fig. 5b). Neither digitoxigenin nor digitoxin, however, had any effect on the initial rate of $\mathrm{K}^{+}$ transport in the presence of free amphotericin $\mathrm{B}$ alone (figs. 5a and 5b). In addition, hydrocortisone succinate had no effect on the rates of cation fluxes, whether used with free or liposome-incorporated amphotericin B. On the other hand, the initial rates of $\mathrm{K}^{+}$and $\mathrm{Na}^{+}$transport were too low to be measured at the relatively low molar ratios of nystatin to liposomal lipids of 2.5/1000 and 3.0/1000 for phosphati- dylcholine and cholesterol-phosphatidylcholine liposomes, respectively. Furthermore, comparably low concentrations of free nystatin failed to show any measurable effect on $\mathrm{K}^{+}$release.

\section{Discussion}

The degree of amphotericin B or nystatin incorporation into liposomes appeared to depend on the lipid composition of the liposomes. The amount of amphotericin B or nystatin incorporated into liposomes containing cholesterol was about $11 \%$ higher than into liposomes lacking cholesterol. This could be explained in the light of their different affinities for sterols in biological and model membranes $(2-4,31$, 32 ). The relatively low percentage of nystatin, as compared with amphotericin B, incorporated into liposomes may be due to the combined effects of its relatively lower partition coefficient in decane and in the erythrocyte membrane (33) and the lower affinity and effectiveness with which is binds $(31,32)$ to membrane cholesterol. Moreover, using size distribution analysis with a Coulter Counter, we showed that the formation of liposomes from both phosphatidylcholine and cholesterol led to an $8.3 \%$ reduction in the 

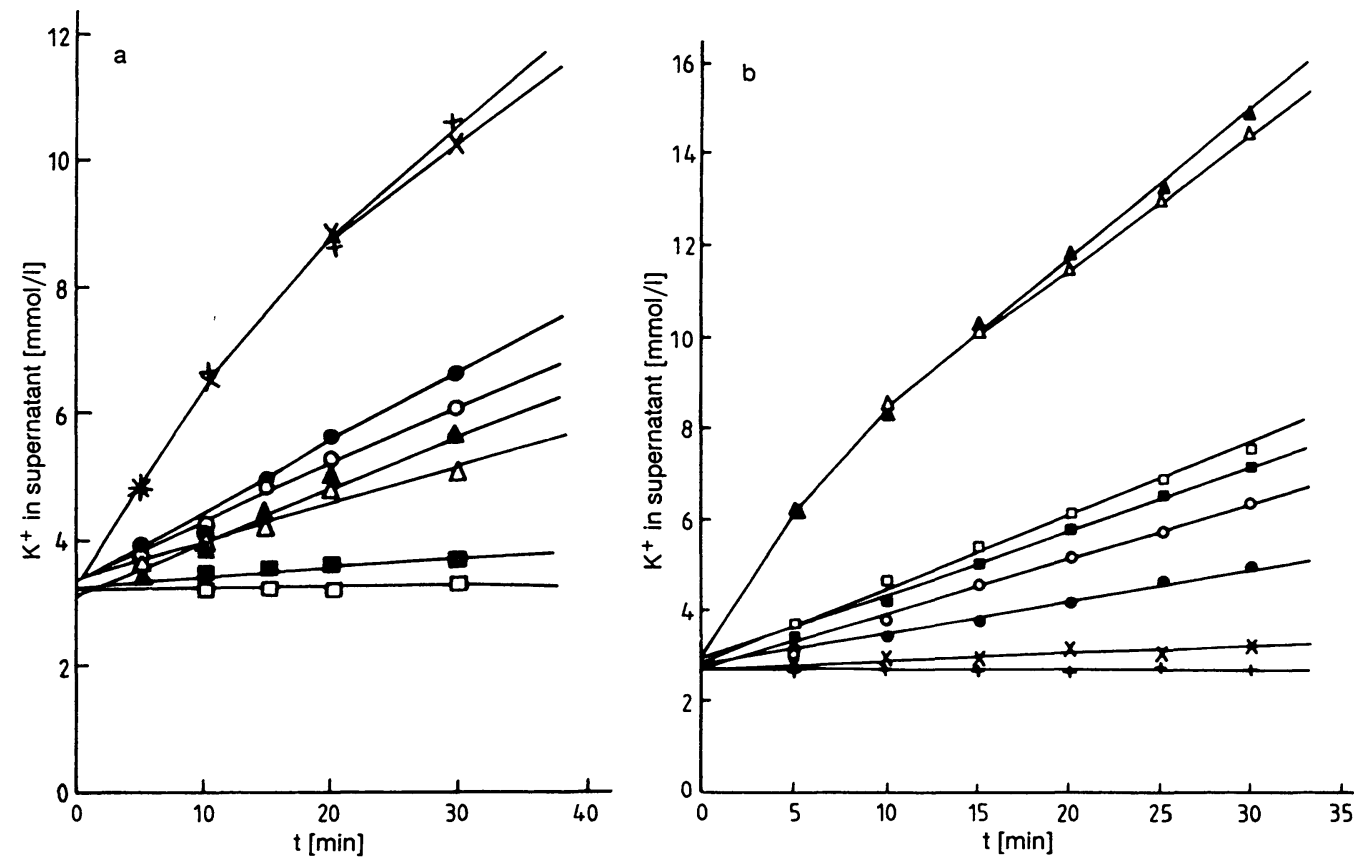

Fig. 5. a. Effect of digitoxigenin on the rate of $\mathrm{K}^{+}$efflux. Washed human erythrocytes were incubated at $37^{\circ} \mathrm{C}$ with $0 \mu \mathrm{mol} / 1$ () or $150 \mu \mathrm{mol} / 1$ digitoxigenin ( $\square$ ); amphotericin B, in $1000 \mu \mathrm{mol}$ of liposomal cholesterol and phosphatidylcholine (molar ratio $3: 7$ ) at a concentration of $8.1 \mu \mathrm{mol} / 1(\Delta)$, plus $150 \mu \mathrm{mol} / 1$ digitoxigenin $(\Delta)$ in the final suspension; amphotericin B in $1000 \mu \mathrm{mol}$ of liposomal phosphatidylcholine, at a concentration of $7.4 \mu \mathrm{mol} / 1$ (o), plus $150 \mu \mathrm{mol} / 1$ digitoxigenin (๑) in the final suspension; $8.1 \mu \mathrm{mol} / 1$ free amphotericin $\mathrm{B}(+)$ plus $150 \mu \mathrm{mol} / 1$ digitoxigenin (x).

b. Effect of digitoxin on the rate of $\mathrm{K}^{+}$efflux. Washed human erythrocytes were incubated at $37^{\circ} \mathrm{C}$ with $0 \mu \mathrm{mol} / 1(+)$ or $150 \mu \mathrm{mol} / 1$ (x) digitoxin; amphotericin B in $1000 \mu \mathrm{mol}$ of liposomal cholesterol and phosphatidylcholine (molar ratio $3: 7)$ at a concentration of $8.1 \mu \mathrm{mol} / 1(\bullet)$, plus $150 \mu \mathrm{mol} / 1$ digitoxin (o) in the final suspension; amphotericin $\mathrm{B}$ in 1000 $\mu \mathrm{mol}$ of liposomal phosphatidylcholine at a concentration of $7.4 \mu \mathrm{mol} / \mathrm{l}(\boldsymbol{\square})$, plus $150 \mu \mathrm{mol} / 1$ digitoxin ( $\square$ ) in the final suspension; $7.4 \mu \mathrm{mol} / 1$ free amphotericin $B(\Delta)$ plus $150 \mu \mathrm{mol} / 1$ digitoxin $(\triangle)$. Each point represents the mean of five different studies.

total number of liposomes formed. Nevertheless, the total volume of liposomes containing cholesterol in the range examined was found to be $19 \%$ lower that that of liposomes lacking cholesterol. This indicates a shift toward smaller liposomes and vesicles in the former case. The shift toward smaller liposomes may be explained by the known effect of cholesterol in causing tighter packing of phospholipid molecules rich in unsaturated fatty acids $(34,35)$, thereby allowing tighter packing of individual lamellae.

The significant increase in the total number of amphotericin B particles upon sonication can be accounted for, at least in part, by sonication-induced dispersion of large amphotericin B particles to form smaller ones. Moreover, size distribution analysis showed that amphotericin B incorporation shifts cholesterol-containing liposomes towards a larger size than cholesterol-lacking liposomes. This might be explained mainly by the ability of amphotericin B to complex cholesterol, thereby rendering it unavailable for interaction with neighbouring phospholipid molecules (8); this would cause liposomal lamellae to be less tightly packed than those of cholesterol lacking liposomes.
The effects of liposome-incorporated amphotericin B on the transport profiles of cations across the membrane of the human erythrocyte might be expected to differ from those obtained with free antibiotics (6). The transport of both cations investigated in this work appeared to undergo significant reduction in the presence of liposome-incorporated amphotericin B, when compared with their rates of transport with comparable concentrations of free antibiotic. The more effective reduction of $\mathrm{K}^{+}$release by cholesterol-phosphatidylcholine liposomes containing amphotericin $\mathrm{B}$ was attributed to the relatively tight binding of amphotericin B to the cholesterol available in liposomes. This relatively tight binding effect impedes the exchange of the drug with the erythrocyte membrane, thus reducing the drug's potential to interact with the erythrocyte membrane and consequently to alter its permeability properties, which may lead otherwise to haemolysis. Thus, it appears that the type and nature of lipids constituting the liposomes, used as drug delivery vehicles, play an important role in controlling the rate of drug release and the rate of cation transport (and presumably anion transport) across the human erythrocyte, and possibly other types of mammalian cell membrane. Therefore, the intravenous in- 
jection of cholesterol-phosphatidylcholine liposomes containing amphotericin B may ensure the gradual release of the antibiotic; this would eliminate the need for routine injections of free amphotericin $B$ in small doses over a period of $3-4 \mathrm{~h}(36)$. Moreover, the use of cholesterol-phosphatidylcholine liposomes containing amphotericin should allow the use of a higher single therapeutic dose, while keeping undesirable cation imbalances, and consequently the potential occurrence of haemolytic anaemia, under control.

On the other hand, when incorporated into either type of liposome, the efficacy of amphotericin B in minimizing the initial rate of cation exchange was reduced in the presence of some free steroid drugs such as digitoxigenin and digitoxin, used clinically in the treatment of congestive heart failure. This effect was attributed to the inhibitory action of these drugs on the $\mathrm{Na}^{+}-\mathrm{K}^{+}$pump (37), allowing $\mathrm{Na}^{+}$and $\mathrm{K}^{+}$ transport to occur freely across the human erythrocyte membrane, thus causing an ionic imbalance. Nevertheless, the initial rate of transport of $\mathrm{K}^{+}$ions did not seem to be affected when these two cardiotonic steroids were mixed with comparable concentrations of free amphotericin B. This indicates that the $\mathrm{Na}^{+}$$\mathrm{K}^{+}$pump continues to be responsive to the action of these cardiotonic steroids in the presence of the low amounts of amphotericin B transferred from liposomes to the erythrocyte membrane, but it may fail to respond in the presence of high concentrations of free antibiotic. This is explained by the fact that free amphotericin B has a high partition coefficient in the erythrocyte membrane and causes a significant perturbation of its normal fluidity (33), which is necessary for the $\mathrm{Na}^{+}-\mathrm{K}^{+}$pump to assume its usual conformation and activity, including its ability to bind cardiotonic steroids.

Hydrocortisone succinate is another steroid given with amphotericin B to reduce the febrile reactions that may follow intravenous injection of the latter (1). Although it contains the cholestane ring structure, hydrocortisone succinate was found to have no effect on the rate of $\mathrm{K}^{+}$transport, when used in conjunction with free or with liposome-incorporated amphotericin B. Moreover, it did not seem to complex with amphotericin $\mathrm{B}$, as cholesterol did $(2-4)$, as evidenced by the UV absorption spectra obtained for amphotericin B in the presence and absence of the drug (UV absorption spectra not shown). This is presumably due to the absence of a hydroxyl group at $C_{3}$ in the ring structure of hydrocortisone succinate.

It thus appears that the mechanism by which cell damage and toxicity by amphotericin B, as applied to human erythrocytes and possibly other types of mammalian cells, is mediated by an increased rate of $\mathrm{K}^{+}$and $\mathrm{Na}^{+}$transport. This problem can be approached by:

a) incorporating amphotericin B into liposomes;

b) deriving a method by which specific amounts of amphotericin B can be incorporated in liposomes;

c) devising a method which allows the efficient removal of un-incorporated antibiotic;

d) selecting the size distribution of amphotericin B particles;

e) incorporating cholesterol as a constituent of the liposomes;

f) selecting the optimal concentration of amphotericin $B$ which has a minimal effect on membrane fluidity and consequently on the activity of transport proteins, such as the $\mathrm{Na}^{+}-\mathrm{K}^{+}$pump; and

g) taking into consideration the effects of any other drugs taken simultaneously with amphotericin B.

Incorporation of amphotericin B in liposomes may result, however, in a reduction in the therapeutic efficacy of the drug.

\section{Acknowledgement}

The author would like to thank $H$. A. Gambo and $A$. A. Awad for their technical assistance and the Authority of King Khalid University Hospital for providing the blood. This work was supported by grant No. Bio/1411/16 from the Research Center of the College of Science, King Saud Unversity.

\section{References}

1. Reynolds, J. E. F. 29th (ed.) (1989) Marindale - The Extra Pharmacopeia, pp. 417-420. The Pharmaceutical Press, London.

2. De Kruijff, B., Gerritsen, W. J., Oerlemans, A., Demel, R A. \& Van Deenen, L. L. M. (1974) Polyene AntibioticSterol Interactions in Membranes of Acholeplasma Laidlawii cells and Lecithin Liposomes. I. Specificity of the Membrane Permeability Changes Induced by the Polyene Antibiotics. Biochim. Biophys. Acta 339, 30-43.
3. De Kruijff, B. \& Demel, R. A. (1974) Polyene AntibioticSterol Interactions in Membranes of Acholeplasma Laidlawii cells and Lecithin Liposomes III. Molecular Structure of the Polyene Antibiotic-Cholesterol Complexes. Biochim. Biophys. Acta 339, 57-70.

4. Teerlink, T., De Kruijff, B. \& Demel, R. A. (1980) The Action of Pimaricin Etruscomycin and Amphotericin B on Liposomes with Varying Sterol Content. Biochim. Biophys. Acta 599, 484-492. 
5. Van Hoogevest, P. \& De Kruijff, B. (1978) Effect of Amphotericin B on Cholesterol-Containing Liposomes of Egg Phosphatidylcholine and Didocosenoyl Phosphatidylcholine. A Refinement of the Model for the Formation of Pores by Amphotericin B in Membranes. Biochim. Biophys. Acta 511, 397-407.

6. Abu-Salah, K. M., Sedrani, S. H., Tobia, A. S. \& Gambo, H. A. (1988) Influence of Amphotericin B on the Transport of Phosphate, Sulphate and Potassium Ions Across the Human Erythrocyte Membrane. Acta Haematologica 79, $77-80$.

7. Abu-Salah, K. M. (1984) Some Effects of Nystatin on Phosphate and Sulphate Transport Across the Membrane of the Human Erythrocyte. Med. Sci. Res. (UK) 12, 526527.

8. De Kruijff, B. Gerritsen, W. J., Oerlemans, A., Van Dijck, P. W. M. \& Van Deenen, L. L. M. (1974) Polyene Antibiotic-Sterol Interactions in Membranes of Acholeplasma Laidlawii cells and Lecithin Liposomes. III. Temperature Dependence of the Polyene Antibiotic-Sterol Complex Formation. Biochim. Biophys. Acta 339, 44-56.

9. Andreoli, T. E. (1974) The Structure and Function of Amphotericin B-Cholesterol Pores in Lipid Bilayer Membranes. Ann. N. Y. Acad. Sci. 235, 448-468.

10. Kinsky, S. C. (1970) Antibiotic Interactions with Model Membranes. Annu. Rev. Pharmacol. 10, 119-142.

11. Ryler, J. F., Wilson, R. G., Gravestock, M. B. \& Poyser, J. F. (1981) Experimental Approaches to Antifungal Chemotherapy. Adv. Pharmacol. Chemother. 18, 49-176.

12. Lopez-Berestein, G., Bodey, G. P., Frankel, L. S. \& Mehta, K. (1987) Treatment of Hepatosplenic Fungal Infections with Liposomal-Amphotericin B. J. Clin. Oncol. 5, 310317.

13. Lopez-Berestein, G., Fainstein, V., Hopfer, R. L., Mehta, K., Sullivan, M. P., Keating, M., Rosenblum, M. G., Mehta, R., Luna, M., Hersh, E. M., Reuben, J., Juliano, R. L. \& Body, G. P. 1985. Liposomal-Amphotericin B for the Treatment of Systemic Fungal Infections in Patients with Cancer: A Prelimenary report. J. Infect. Dis. 4, 704710.

14. Degregorio, M. W., Lee, W. M. F., Linker, C. A., Jacobs, R. A. \& Ries, C. A. (1982) Fungal Infections in Patients with Acute Leukemia. Am. J. Med. 73, 543-548.

15. Gold, J. W. M. (1984) Opportunistic Fungal Infections in Patients with Neoplastic Disease. Am. J. Med. 76, 458463.

16. New, R. R. C., Chance, M. L. \& Heath, S. (1981) Antileishmanial Activity of Amphotericin and other Antifungal Agents Entrapped in Liposomes. J. Antimicrob. Chemother. $8,371-381$.

17. Graybill, J. R. Craven, P. C., Taylor, R. L., Williams, D. L. \& Magee, W. E. (1982) Treatment of Murine Cryptococcosis with Liposome-Associated Amphotericin B. J. Infect. Dis. $145,748-752$.

18. Taylor, R. L., Williams, D. M., Craven, P. C., Graybill, J. R., Drutz, D. J. \& Magee, W. E. (1982) Amphotericin B in Liposomes: a Novel Therapy of Histoplasmosis. Am. Rev. Respir. Dis. 125, 610-611.

19. Lopez-Berestein, G., Hopfer, R. L., Mehta, R., Mehta, K., Hersh, E. M. \& Juliano, R. L. (1984) Liposomes-Encapsulated Amphotericin B for the Treatment of Dessiminated Candidiasis in Neutropenic Mice. J. Infect. Dis. 150, $278-$ 283.
20. Lopez-Berestein, G., McQueen, T. \& Mehta, K. (1985) Protective Effect of Liposomal-Amphotericin B Against Candida Infection in Mice. Cancer Drug Delivery 2: 183189.

21. Batzri, S. \& Korn, E. E. 1973. Single Bilayer Liposomes Prepared without Sonication. Biochim. Biophys. Acta 298, 1015-1019.

22. Bangham, A. D., Hill, M. W. \& Miller, N. G. A. (1974) Preparation and use of Liposomes as Models of Biological Membranes. Methods Membr. Biol. 1, 1-68.

23. Szoka, F. \& Papahadjopoulos, D. (1980) Comparative Properties and Methods of Preparation of Lipid Vesicles (Liposomes). Annu. Rev. Biophys. Bioeng. 9, 465-506.

24. New, R. R. C., Chance, M. L., Thomas, S. C. \& Peters, W. (1978) Antileishmanial Activity of Antimonials Entrapped in Liposomes. Nature (London) 272, 55- 56.

25. Prison, P., Steiger, R. \& Trouet, A. (1982) The Disposition of Free and Liposomally Encapsulated Antimalarial Primaquine in Mice. Biochem. Pharmacol. 31, 3501-3507.

26. Gabizon, A., Dagan, A., Goren, D., Barenholtz, Y. \& Fuks, Z. (1982) Liposomes as in vivo Carriers of Adriamycin: Reduced Cardiac Uptake and Preserved Antitumour Activity in Mice. Cancer Res. 42, 4734-4739.

27. Lopez-Berestein, G. (1987) Liposomes as Carriers of Antimicrobial agents. Antimicrob. Chemother. 31, 675-678.

28. Feigin, A., Bayelousova, I., Yakhimovich, R., Vasilyevskaya, V. \& Tereshin, I. (1979) Role of the Number and Position of Double Bonds in the Tetracyclic Nucleus of Sterols on the Interaction with Polyene Antibiotics. Biofizika 24: $330-331$.

29. Chen, P. S., Toribara, T. Y. \& Warner, H. (1956) Microdetermination of Phosphorus. Anal. Chem. 28, 1751-1756

30. Leffler, H. H. \& McDougald, C. H. (1963) Estimation of Cholesterol in Serum. Am. J. Clin. Pathol. 39, 311-315.

31. Demel, R. A., Grombag, F. J. L., Van Deenen, L. L. M. \& Kinsky, S. C. (1968) Interaction of Polyene Antibiotics with Single and Mixed Lipid Monomolecular Layers. Biochim. Biophys. Acta 150, 1-14.

32. Norman, A. W., Demel, R. A., Dekruijff, B., Geurts Van Kessel, W. S. M. \& Van Deenen, L. L. M. (1972) Studies on the Biological Properties of Polyene Antibiotics: Comparison of other Polyenes with Filipin in their Ability to Interact Specifically with Sterol. Biochim. Biophys. Acta $290,1-14$

33. Abu-Salah, K. M. (1991) Perturbation of the Fluidity of the Erythrocyte Membrane with Iorophoric Antibiotics and Lipophilic Anaesthetics. Biochem. Pharmacol. 42, 19471951.

34. Ladbrooke, B. D. \& Chapman, D. (1969) Thermal Analysis of Lipids, Proteins and Biological Membranes. Chem. Phys. Lip. 3, 304-356.

35. Giraud, F., Claret, M., Bruckdorfer, R. \& Chilley, B. (1981) The Effects of Membrane Lipid Order and Cholesterol on the Internal and Internal Cationic Sites of the $\mathrm{Na}^{+}-\mathrm{K}^{+}$ Pump in Erythrocytes. Biochim. Biophys. Acta 647, 249258.

36. The Pharmaceutical Society of Great Britain (ed.) (1979) The Pharmaceutical Codex; 11th ed., pp. 39-41, Pharmaceutical Press, London.

37. Ahmed, K., Rohrer, D. C., Fullerton, D. S., Deffo, T., Kitatsuji, E. \& Form, A. H. L. (1983) Interaction of $\left(\mathrm{Na}^{+}, \mathrm{K}^{+}\right)$-ATPase and Digitalis Genins: A General Model for Inhibitory Activity. J. Biol. Chem. 258, 8092-8097.

Dr. Khalid M. Abu-Salah
Associate Professor of Biochemistry
College of Science
King Saud University
P. O. Box 2455
Riyadh 11451
Saudi Arabia

Dr. Khalid M. Abu-Salah

College of Science

King Saud University

Riyadh 11451

Saudi Arabia

Eur. J. Clin. Chem. Clin. Biochem. / Vol. 30, 1992 / No. 11 